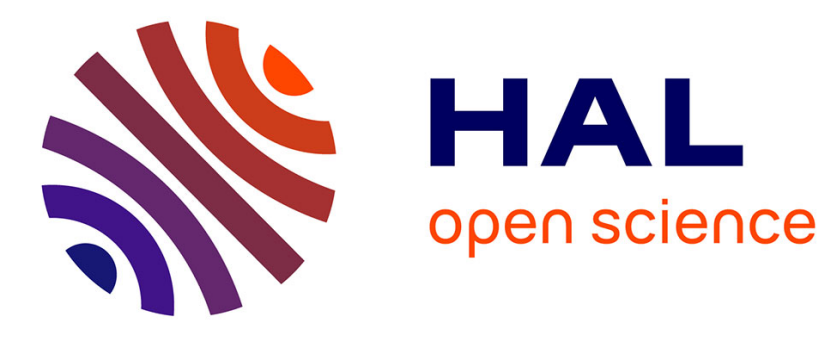

\title{
Fast and high quality topology-aware task mapping
}

\author{
Mehmet Deveci, Kamer Kaya, Bora Uçar, Umit V. Catalyurek
}

\section{To cite this version:}

Mehmet Deveci, Kamer Kaya, Bora Uçar, Umit V. Catalyurek. Fast and high quality topology-aware task mapping. 29th IEEE International Parallel \& Distributed Processing Symposium, May 2015, Hyderabad, India. hal-01159677

\section{HAL Id: hal-01159677 \\ https://hal.inria.fr/hal-01159677}

Submitted on 3 Jun 2015

HAL is a multi-disciplinary open access archive for the deposit and dissemination of scientific research documents, whether they are published or not. The documents may come from teaching and research institutions in France or abroad, or from public or private research centers.
L'archive ouverte pluridisciplinaire HAL, est destinée au dépôt et à la diffusion de documents scientifiques de niveau recherche, publiés ou non, émanant des établissements d'enseignement et de recherche français ou étrangers, des laboratoires publics ou privés. 


\title{
Fast and high quality topology-aware task mapping
}

\author{
Mehmet Deveci*, Kamer Kaya $^{\dagger *}$, Bora Uçar ${ }^{\ddagger}$, Ümit V. Çatalyürek* \\ *Biomedical Informatics, The Ohio State University, Columbus, OH \\ $\{$ mdeveci,kamer,umit\}@bmi.osu.edu \\ ${ }^{\dagger}$ Computer Science and Engineering, Sabancı University, Istanbul, Turkey \\ kaya@ sabanciuniv.edu \\ ${ }^{\ddagger}$ CNRS and LIP (UMR 5668), ENS, Lyon, France \\ bora.ucar@ens-lyon.fr
}

\begin{abstract}
Considering the large number of processors and the size of the interconnection networks on exascalecapable supercomputers, mapping concurrently executable and communicating tasks of an application is a complex problem that needs to be dealt with care. For parallel applications, the communication overhead can be a significant bottleneck on scalability. Topology-aware task-mapping methods that map the tasks to the processors (i.e., cores) by exploiting the underlying network information are very effective to avoid, or at worst bend, this limitation. We propose novel, efficient, and effective task mapping algorithms employing a graph model. The experiments show that the methods are faster than the existing approaches proposed for the same task, and on 4096 processors, the algorithms improve the communication hops and link contentions by $16 \%$ and $32 \%$, respectively, on the average. In addition, they improve the average execution time of a parallel SpMV kernel and a communication-only application by $9 \%$ and $14 \%$, respectively.

Keywords-Task mapping, communication graph, partitioning
\end{abstract}

\section{INTRODUCTION}

For parallel computing, task mapping has a significant impact on the performance, especially when supercomputers with hundreds of thousands or millions of processors shoulder the execution. It is usually the case that the communication pattern between the tasks has already been designed to minimize possible performance bottlenecks, such as high number of messages or communication volume, via tools such as graph and hypergraph partitioners, e.g., [1], [2], [3], [4], [5]. However, this effort alone is not sufficient, since the mapping-based metrics such as the maximum link congestion and the total number of hops the messages travel in the network can also be significant bottlenecks on the performance. This is especially true for today's supercomputers with large-diameter interconnection networks, and concurrent and non-uniform job submissions yielding sparse and wide-spread processor allocations for parallel applications. There exist various studies in the literature which analyze the impact of task-to-processor mappings on the parallel performance [6], [7], [8], [9] and report significant speedups, e.g., 1.64X [10], just with an improved mapping.

There are two main research directions for mapping. The first one focuses on block-based processor allocations, e.g., the ones on IBM BlueGene [8], [10], [11], [12], [13]. The second direction focuses on sparse allocations in which the allocated processors are not contiguous within the network [14], [15]. This direction is more general: sparse allocations have been used in various parallel systems, and the mapping algorithms based on this model can also be used for the block-based model. In this work, we follow the second direction.

The problem of finding an optimal task-to-processor mapping is NP-complete [15], and various heuristics have been proposed [16], [17], [18], [19]. Many of these heuristics use graphs and related combinatorial structures to model the task interactions as well as the network topologies. For example, the open-source mapping library LibTopoMap [15] uses a task graph and network topology information. The task graph is first partitioned into the number of allocated nodes, and various graph-based algorithms are used to map the tasks to the allocated processors. Other libraries such as JOSTLE [20] and Scotch [1] also exist. These two libraries apply simultaneous partitioning and mapping of the task and topology graphs.

A good mapping algorithm must be able to provide highquality task-to-processor mappings. It also needs to be efficient in order not to intervene the supercomputer's performance. We follow these two important criteria and propose novel, very efficient, refinement-based mapping algorithms. We show that they can produce high-quality mappings w.r.t. the topologyrelated metrics such as the average link congestion and the total hops the messages take. We compare the performance of the proposed algorithms with that of LibTopoMap and Scotch. The experiments on a supercomputer with a 3D torus network and 4096 processors show that the algorithms can improve the weighted hop and the maximum congestion (that will be described below) by $16 \%$ and $32 \%$ on the average, respectively, compared to the default mapping. These metric improvements yield a $43 \%$ performance improvement on one case for a synthetic, communication-only application (Figure 4b-PATOH) and a $23 \%$ improvement on the performance of a sparse-matrix vector multiplication (SpMV) kernel (Figure 5-METIS). Overall, with 4096 and 8192 processors, they improve the performance of a parallel SpMV kernel and a communicationonly application by $9 \%$ and $14 \%$, respectively (Table I).

The organization of the paper is as follows. In Section II, the background for the topology-aware task mapping is given. We also summarize the related work and the target architecture in this section. In Section III, we present three mapping algorithms minimizing various metrics. Section IV presents the experimental results, and Section V concludes the paper. 


\section{BACKGROUND}

Let $G_{t}=\left(V_{t}, E_{t}\right)$ be a directed MPI task graph, where $V_{t}$ is the task set, and $E_{t}$ is the edge set modeling task-to-task communications, i.e., $\left(t_{1}, t_{2}\right) \in E_{t}$ if and only if $t_{1} \in V_{t}$ sends a message to $t_{2} \in V_{t}$. Let $G_{m}=\left(V_{m}, E_{m}\right)$ be the network topology graph where $V_{m}$ is the set of computing nodes equipped with many processors/cores, and $E_{m}$ is the edge set modeling the physical communication links between the nodes, i.e., $\left(m_{1}, m_{2}\right) \in E_{m}$ if and only if $m_{1} \in V_{m}$ and $m_{2} \in V_{m}$ have a link in between. Let $V_{a} \subseteq V_{m}$ be the set of computing nodes reserved for the application. The topology-aware mapping problem can be defined as finding a mapping function $\left(\Gamma: V_{t} \rightarrow V_{a}\right)$ that minimizes the parallel execution time. For a task set $S \subseteq V_{t}$, we use $\Gamma[S]$ to denote the node set to which the tasks in $S$ are mapped to.

There are two well-received metrics to model the network communication overhead. The total hop count (TH), which is the total length of paths taken by communication packets, is a latency-based metric. The maximum message congestion (MMC), which is the maximum number of messages sent across a link. We assume that the messages are not split and sent through only a single path via static routing.

Let $\Gamma$ be a given mapping where $m_{1}=\Gamma\left(t_{1}\right)$ if and only if $t_{1}$ is assigned to one of node $m_{1}$ 's processors. Then

$$
\mathrm{TH}(\Gamma)=\sum_{\left(t_{1}, t_{2}\right) \in E_{t}} \text { dilation }\left(t_{1}, t_{2}\right),
$$

where dilation $\left(t_{1}, t_{2}\right)=\operatorname{SPL}\left(\Gamma\left(t_{1}\right), \Gamma\left(t_{2}\right), G_{m}\right)$ which is the shortest-path length between $\Gamma\left(t_{1}\right) \in V_{m}$ and $\Gamma\left(t_{2}\right) \in V_{m}$.

Let the congestion on a network link $e \in E_{m}$ be

$$
\text { Congestion }(e)=\sum_{\left(t_{1}, t_{2}\right) \in E_{t}} \operatorname{inSP}\left(e, \Gamma\left(t_{1}\right), \Gamma\left(t_{2}\right), G_{m}\right),
$$

where, inSP $=1$ if and only if $e$ is on the shortest path between $\Gamma\left(t_{1}\right)$ and $\Gamma\left(t_{2}\right)$, and 0 otherwise. Therefore, a link's congestion is equal to the number of messages that passes through it. Hence, the maximum message congestion is

$$
\operatorname{MMC}(\Gamma)=\max _{e \in E_{m}}\{\text { Congestion }(e)\} .
$$

The above metrics assume unit communication costs and link capacities. In order to handle heterogeneous costs and bandwidths, the task and topology graph models are extended as follows. Each edge $e^{\prime} \in E_{t}$ in the task graph is associated with a cost $c\left(e^{\prime}\right)$ that corresponds to the communication volume sent/received between the tasks. Similarly, each link $e \in E_{m}$ in the topology graph is associated with a communication capacity $b w(e)$ that corresponds to the link bandwidth. Moreover, for further heterogeneity support, each node $m \in V_{m}$ is associated with a computation capacity $w(m)$ that corresponds to the number of available (allocated) processors in $m$. All the nodes that are not in $V_{a}$ have zero capacity. Based on these attributes on the edges, the weighted hop (WH) metric, i.e., the total number of hops taken by each packet, is

$$
\mathrm{WH}(\Gamma)=\sum_{\left(t_{1}, t_{2}\right) \in E_{t}} \text { dilation }\left(t_{1}, t_{2}\right) c\left(t_{1}, t_{2}\right) .
$$

We define the volume congestion (VC) of a link $e \in E_{m}$ as

$$
\mathrm{VC}(e)=\frac{\sum_{\left(t_{1}, t_{2}\right) \in E_{t}} \operatorname{inSP}\left(e, \Gamma\left(t_{1}\right), \Gamma\left(t_{2}\right), G_{m}\right) c\left(t_{1}, t_{2}\right)}{b w(e)}
$$

which is the ratio between the overall volume of the communication passing from $e$ and its bandwidth. Then, the max volume congestion (MC) metric is the congestion of the bottleneck link in the network with the maximum $\mathrm{VC}$ value.

A communication-bounded parallel application can be either latency-bounded or bandwidth-bounded depending on the communication pattern. For example, applications with frequent communication steps and small messages are likely to be latency-bounded, while those with larger messages are likely to be bandwidth-bounded. For latency-bounded applications, TH and $\mathrm{WH}$ metrics can better correlate with the communication overhead. On the contrary, MMC and MC are better choices for the bandwidth-bounded applications. Depending on the communication pattern, it might be better to minimize MC (MMC) in the expense of an increase on WH (TH), or vice versa. However, it is indeed difficult to find a consensus between these metrics. Therefore, here we define average message congestion (AMC) and average congestion (AC) metrics to account for both number of hops and congestion. Let $E_{m}^{t}$ be the set of the links that are used throughout the execution of the parallel application. The message and volume based average link congestions over the used links can be defined as

$$
\begin{gathered}
\operatorname{AMC}(\Gamma)=\frac{\sum_{e \in E_{m}} \text { Congestion }(e)}{\left|E_{m}^{t}\right|}, \\
\operatorname{AC}(\Gamma)=\frac{\sum_{e \in E_{m}} \operatorname{VC}(e)}{\left|E_{m}^{t}\right|}
\end{gathered}
$$

Since $\mathrm{TH}=\sum_{e \in E_{m}}$ Congestion(e), AMC is the ratio of TH to the total number of links used during the application. Similarly, AC is related to the ratio of WH to the number of links used during the execution (they are equal only when the communication links have unit bandwidths).

The communication during the execution is a real-time process that can easily be affected by many outside factors (e.g., network traffic and overhead from competing jobs). Hence, the theoretical metrics given above can only approximate the actual overhead. In Section IV-E, we will discuss and evaluate the interplay of these metrics on the performance.

\section{A. Related Work}

Existing mapping algorithms can be divided into two classes: single-phase and two-phase methods. The algorithms in the former class perform simultaneous partitioning and mapping using the task and topology graphs, whereas those in the latter partition the task graph in the first phase and map the parts to the processors in the second one.

Pellegrini and Roman [21] proposed a single-phase recursive-bipartitioning algorithm for various topologies. Wallshaw and Cross [20] proposed a multilevel partitioning algorithm which performs mapping in the initial partitioning and refinement phases. 
The two-phase mapping methods make an abstraction of the partitioning phase and work with a pre-partitioned task set. These studies can be divided into two based on the taskdependency model and network topology. The first set adapts geometric task-interaction models, e.g., [22], [23] on IBM's BlueGene systems with block-based node allocations and on sparse allocations [14]. Still, most of the work focuses on the connectivity-based models, specifically graph models. This problem is shown to be NP-complete [15], [24], and many heuristics exist in the literature, e.g., [16], [17], [18], [19]. In this work, we investigate a two-phase graph-based approach.

\section{B. The architecture}

Six of the top ten supercomputers in the Top500 list (June 2014), have torus networks (one 3D, four 5D, one 6D). In this work, we target NERSC's Hopper supercomputer with Cray XE6's 3D torus. The network has Gemini routers directly connected to two computing nodes. Each router is associated with a coordinate on $x, y$, and $z$ dimensions, and connected to all six neighbor routers. The torus network provides wrap-arounds, and the messages between the nodes are statically routed following the shortest paths. The network links have different bandwidth values on various dimensions.

On Cray systems, the scheduler allocates a non-contiguous set of nodes for each job [25]. Although it attempts to assign nearby nodes, no locality guarantee is provided. The topology information, e.g., the routers' coordinates and connections, as well as link bandwidths, can be captured using system calls (rtr), and a static topology graph can be obtained. During runtime, each MPI process can obtain its node id, and the vertices in the topology graph can be associated with the computational units.

In Hopper, the network latencies for the nearest and farthest node pairs are $1.27 \mu \mathrm{s}$ and $3.88 \mu \mathrm{s}$, respectively. The link bandwidths vary from 4.68 to $9.38 \mathrm{~GB} / \mathrm{sec}$. Ideally, reducing the hop counts between the communicating tasks lowers the overhead. But when there are many communicating tasks, a link can be congested due to the communication pattern, which might cause communication stalls and harm the performance. Still, thanks to static routing, the congestion can be measured and optimized accurately.

\section{FASt And High QuAlity MapPing Algorithms}

We propose three mapping algorithms to minimize WH and MC. Here we will describe these algorithms. Their adaptation for $\mathrm{TH}$ and $\mathrm{MMC}$ is trivial. Among them, the ones that minimize WH can be applied to various topologies, whereas those minimizing $\mathrm{MC}$ require static routing.

\section{A. A Greedy mapping algorithm}

The first algorithm Greedy Mapping given in Algorithm 1 finds a mapping $\Gamma: V_{t} \rightarrow V_{m}$ to minimize WH. It uses the task graph $G_{t}$ and the topology graph $G_{m}$. The algorithm is similar to greedy graph growing, and initially maps $N_{B F S}$ seed task vertices to the nodes. It assumes a symmetric $G_{t}$ while finding the neighbors of a given task since $\mathrm{WH}$ is an undirected metric, i.e., the number of hops between $m_{1}$ and $m_{2}$ is the same regardless of direction.

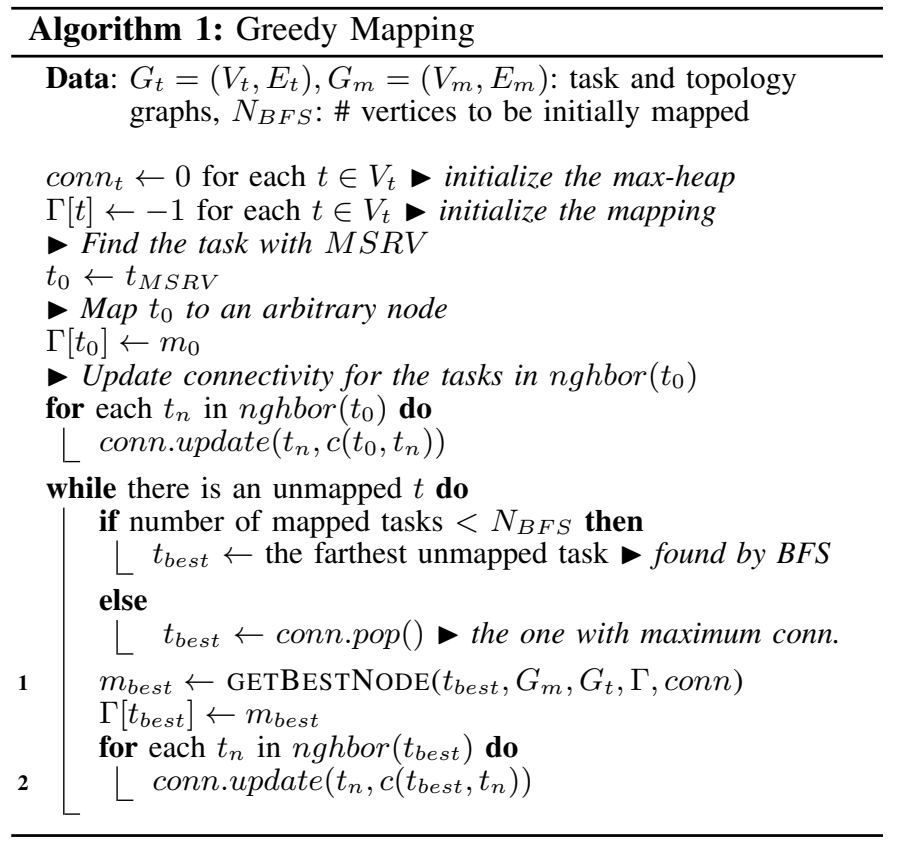

Throughout the algorithm, the total connectivity of each task to the mapped ones are stored in a heap conn. The algorithm first maps the task $t_{M S R V}$ with the maximum send-receive communication volume to an arbitrary node. Until all tasks are mapped, the algorithm gets an unmapped task from conn after all the $N_{B F S}$ seeds are mapped. Otherwise, the farthest task to the set of mapped tasks is found by a breadth-first search (BFS) on $G_{t}$ where all the mapped tasks are assumed to be at level 0 of the BFS. Ties are broken in the favor of the task with a higher communication volume. If $G_{t}$ is disconnected, a task with the maximum communication volume from one of the disconnected components is chosen. Once $t_{\text {best }}$ is found, its best node is obtained by GETBESTNODE. If $t_{\text {best }}$ is connected to none of the mapped tasks, GETBESTNODE performs a BFS on $G_{m}$ to return one of the farthest allocated nodes to the set of the non-empty nodes, i.e., the ones with a mapped task. Otherwise, if $t_{\text {best }}$ is connected to at least one of the mapped tasks, a BFS on $G_{m}$ is performed from the nodes to whom one of the $n g h b o r\left(t_{\text {best }}\right)$ is mapped (again assuming these nodes are at level 0 ). As an early exit mechanism, a BFS stops when the empty nodes (without a mapped task) are found at a BFS level. Then among these empty nodes, the one with the minimum WH overhead is returned. Therefore, the algorithm performs multiple BFS executions on $G_{t}$ and $G_{m}$.

For simplicity, the description above assumes one-to-one task-to-node mapping, i.e., $\left|V_{t}\right|=\left|V_{a}\right|$. In reality, each node has multiple processors, so multiple tasks can be assigned to a single node. These cases can be addressed by using the computation loads and capacities, and modifying GETBESTNODE so that it returns only a node with some free capacity. Another common solution is using traditional graph partitioning as a preprocessing step to reduce the number of tasks to the number 
of the allocated nodes while minimizing the edge-cut [15] We follow this approach and use METIS [3] to partition $G_{t}$ into $\left|V_{a}\right|$ nodes, where the target part weights are the number of available processors on each node in $V_{a}$. Since graph partitioning algorithms do not always obtain a perfect balance, as a post processing, we fix the balance with a small sacrifice on the edge-cut metric via a single Fiduccia-Mattheyses (FM) iteration [26]. When the number of processors in the nodes are not uniform, we map the groups of tasks with different weights at the beginning of the greedy mapping since their nodes are almost decided due their uniqueness.

In the algorithm, $N_{B F S}$ controls the number of initial seed mappings. A large $N_{B F S}$ distributes the loosely connected components of the task graph to the nodes that are farther from each other. However, this will not work well for the task graphs with a low diameter. In our implementation, we use $N_{B F S} \in\{0,1\}$ to generate two different mappings and return the one with the lower WH.

The complexity of the algorithm is dominated by the operations at lines 1 and 2. Each update of the heap (line 2) takes $\mathcal{O}\left(\log \left|V_{t}\right|\right)$ time, and this line is executed at most $\left|E_{t}\right|$ times, yielding $\mathcal{O}\left(\left|E_{t}\right| \log \left|V_{t}\right|\right)$. The BFS operation in GETBESTNODE has $\mathcal{O}\left(\left|E_{m}\right|\right)$ cost, yielding an overall complexity of $\mathcal{O}\left(\left|V_{t}\right|\left|E_{m}\right|\right)$. For a task $t_{\text {best }}$ and a candidate node at the last level of the BFS performed in GETBESTNODE, the cost of computing the change on $\mathrm{WH}$ is proportional to the number of edges of $t_{\text {best }}$ (the hop count between two arbitrary nodes can be found in $\mathcal{O}(1)$, since $G_{m}$ 's are regular graphs). Since there are at most $\left|V_{a}\right|=\left|V_{t}\right|$ candidate nodes and $\left|E_{t}\right|$ edges, the complexity of this part throughout the algorithm is $\mathcal{O}\left(\left|V_{t}\right|\left|E_{t}\right|\right)$. Therefore, the complexity of Algorithm 1 is $\mathcal{O}\left(\left|V_{t}\right|\left(\left|E_{m}\right|+\left|E_{t}\right|\right)\right)$-in practice it runs faster thanks to the early exits in GETBESTNODE BFSs.

\section{B. A Refinement algorithm for the weighted hop}

Algorithm 1 is our main algorithm and the other two will refine its mapping. Even after its execution, it is possible to improve WH via further refinement. We have implemented a Kernighan-Lin [27] type algorithm which uses "task swaps" to refine WH (Algorithm 2). It gets a $\Gamma, G_{t}$, and $G_{m}$ as input and modifies $\Gamma$ to lower the WH metric. Similar to greedy mapping, for simplicity, Algorithm 2 assumes that $G_{t}$ is symmetric and $\Gamma$ is a one-to-one mapping between the tasks and nodes.

The algorithm selects a pair of task vertices and swaps them to improve $\mathrm{WH}$. The first task $t_{w h}$ is chosen using a maxheap, whHeap, which initially organizes the tasks w.r.t. the WH amount they incur computed by a function TASKWHOPS function (line 1). Hence, $t_{w h}$ is the task individually responsible for the largest WH. Choosing the second task for the swap operation is more complicated; a naive approach that considers to swap $t_{w h}$ with all the other tasks requires $\mathcal{O}\left(\left|V_{t}\right|^{2}\right)$ comparisons. In order to avoid this cost, we have implemented a BFS-based task-selection algorithm. A simple observation is that to reduce $\mathrm{WH}, t_{w h}$ needs to move closer to its neighbor tasks. Therefore, we perform a BFS on $G_{m}$ starting from the nodes which have a neighbor of $t_{w h}$, i.e., the

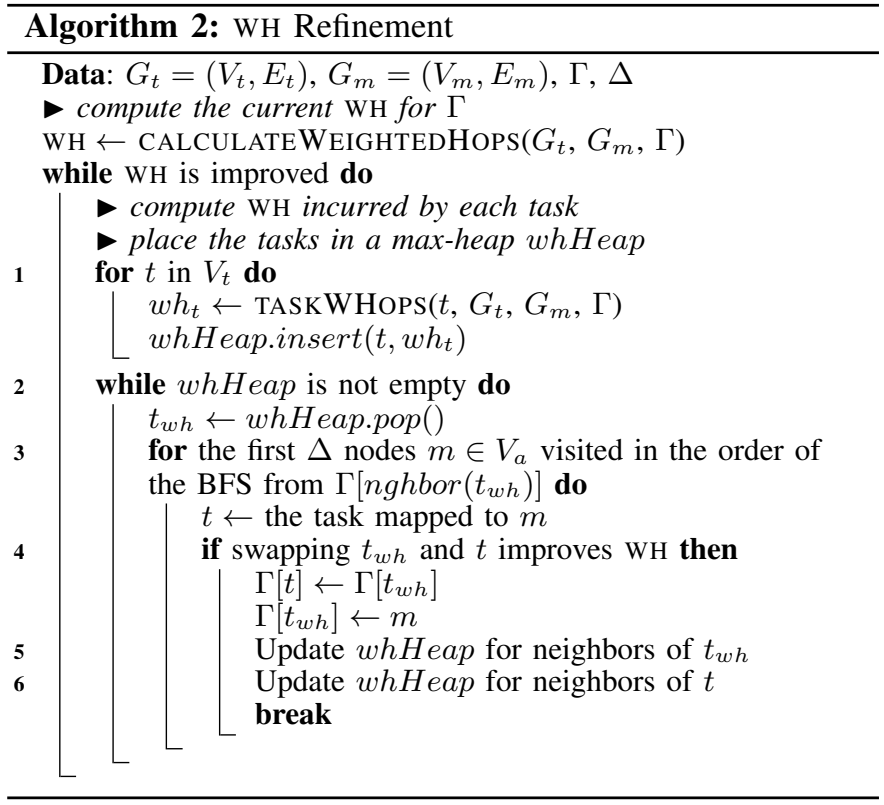

nodes in $\Gamma\left[n g h b o r\left(t_{w h}\right)\right]$ (these are the level 0 nodes of BFS). Whenever a $V_{a}$ node with a the task $t$ is found, the wH value after the potential $\Gamma\left[t_{w h}\right] \leftrightarrow \Gamma[t]$ swap operation is computed. The actual swap is performed as soon as this computation reveals an improvement on WH. Since the likelihood of a WH improvement decreases when we go deeper on the BFS tree, we use an early exit mechanism to avoid a full BFS traversal of $G_{m}$. Here we give an example to clarify the statement, assume $c(e)=1 \forall e \in E_{t}$. If the maximum hop count between $\Gamma\left[t_{w h}\right]$ and $\Gamma\left[n \operatorname{ghbor}\left(t_{w h}\right)\right]$ is $d$ then when $t_{w h}$ is moved to a node after the BFS level $d$, wH incurred by $t_{w h}$ cannot be improved. Furthermore, when we go deeper in the BFS, $t_{w h}$ 's incurred WH value will increase. Even in this case, the overall WH may still be improved due to the reduction of WH incurred by the second task $t$. However, this is less likely to happen considering $\Gamma[t]$ is handpicked for $t_{w h}$ but $\Gamma\left[t_{w h}\right]$ is only a random node for $t$. The early exit mechanism reduces the number of considered swap operations that are unlikely to improve WH. In Algorithm 2, a parameter $\Delta$ is used as an upper bound on this number. If $\Delta$ operations are checked for $t_{w h}$, the algorithm continues with the next whHeap vertex. A refinement pass is completed when whHeap is empty, and the next pass is performed only if there is an improvement in the previous pass.

The complexity of the loop at line 1 is $\mathcal{O}\left(\left|V_{t}\right| \log \left|V_{t}\right|+\left|E_{t}\right|\right)$. The loop at line 2 iterates $\left|V_{t}\right|$ times. The complexity of the BFS operation at line 3 is $\mathcal{O}\left(\left|E_{m}\right|\right)$ and it is also performed $\left|V_{t}\right|$ times. The complexity of the swap operation and the calculation of new $\mathrm{WH}$ at line 4 is proportional to the total number of edges of $t_{w h}$ and $t$ for each candidate node. Since there are at most $\Delta$ candidate nodes for each BFS, the complexity of line 4 for each pass becomes $\mathcal{O}\left(\Delta\left|E_{t}\right|\right)$. Lines 5 and 6 are executed at most once for each vertex and during a single pass, their total cost is $\mathcal{O}\left(\left|E_{t}\right| \log \left|V_{t}\right|\right)$. 
Therefore, the overall complexity of the algorithm becomes, $\mathcal{O}\left(\left|V_{t}\right| \log \left|V_{t}\right|+\left|E_{t}\right|+\left|E_{m}\right|\left|V_{t}\right|+\Delta\left|E_{t}\right|+\left|E_{t}\right| \log \left|V_{t}\right|\right)$. The most dominant factor is the complexity of the BFS operations which is $\mathcal{O}\left(\left|E_{m}\right|\left|V_{t}\right|\right)$. Fortunately, the practical execution time is very low, since we stop after $\Delta=8$ swap candidates. We experimented with other exit mechanisms based on the maximum BFS level instead of the number of swap operations, and the preliminary experiments favored the approach described above. Furthermore, we observed that most of the improvement in $\mathrm{WH}$ is obtained after only a few passes. Hence, in order to be more efficient, we perform a pass only if $\mathrm{WH}$ is improved more than $0.5 \%$ in the previous one.

Similar to that of Algorithm 1, the description above assumes a one-to-one task-to-node mapping and performs the refinement on the node level, i.e., by swapping the vertices representing a group of tasks. With slight modifications, it can perform the refinement on the finer level task vertices or in a multilevel fashion from coarser to finer levels. In our experiments we choose to perform only on the coarser task graphs we obtained after METIS, since with wH-improving swap operations on the finer level, the total internode communication volume can also increase and the performance may decrease. Although this increase can also be tracked during the refinement, we do not want to sacrifice from the efficiency.

\section{A Refinement algorithm for the maximum congestion}

Although Algorithms 1 and 2 significantly improve wH, they can negatively affect MC or MMC, and this can degrade the performance especially for the bandwidth-bounded applications. Therefore, we propose another refinement algorithm (Algorithm 3), which improves the MC metric with minimal $\mathrm{WH}$ damage (adapting this algorithm to refine MMC is trivial). The algorithm can accurately model and minimize MC for the interconnection networks with static routing. We will discuss the required enhancements for the dynamic-routing networks.

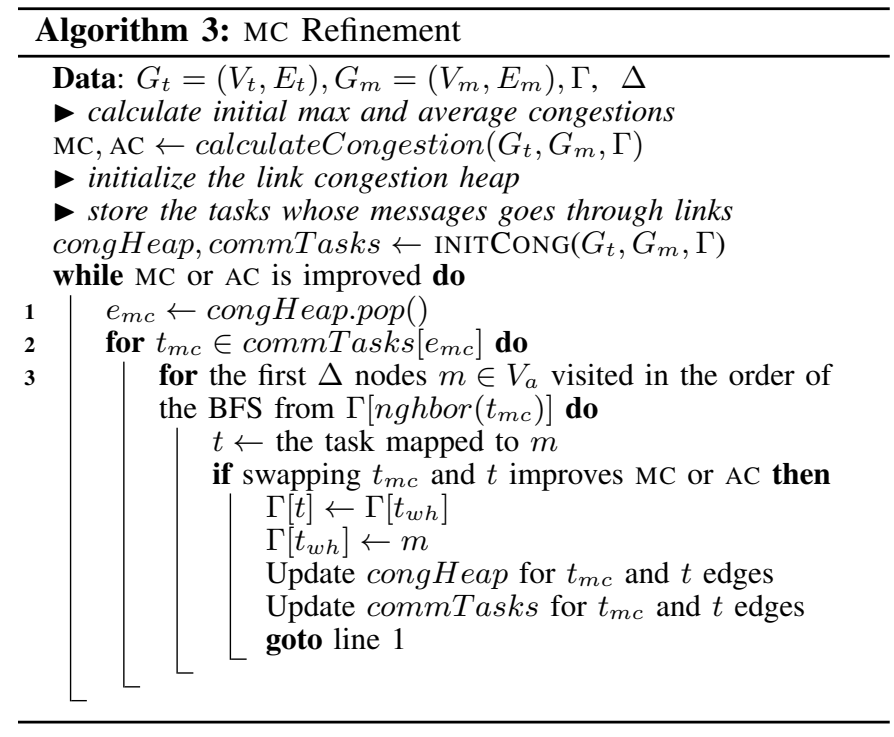

The algorithm gets a $\Gamma, G_{m}$, and $G_{t}$ and modifies $\Gamma$ to find mapping with a better congestion. First, it computes the initial congestion of $\Gamma$, and initializes the congHeap using an INITCONG function. This max-heap stores the topology graph edges w.r.t. their congestion values. The algorithm also initializes commTasks, that is used to query the tasks whose messages go through link e, i.e., commTasks[e]. Since, a message can go at most $D$ (network diameter) hops, the maximum size of commTasks becomes $\left|E_{t}\right| D$, which is manageable since $D$ is not a large number.

After the initialization, the algorithm finds the most congested link $e_{m c}$. Then for each $t_{m c} \in \operatorname{commTasks}\left[e_{m c}\right]$, the node to which $t_{m c}$ will be moved is sought via BFS traversals on $G_{m}$ starting from the nodes $\Gamma\left[n g h b o r\left(t_{m c}\right)\right]$. The second task $t$ to swap is chosen from the tasks of the $V_{a}$ nodes traversed during the BFSs. This BFS order is important to have a minimal damage on WH. For each such candidate node, a virtual swap operation is performed to compute new MC and AC values. As soon as an improvement is detected, the actual swap operation is performed, and the execution continues with the next congested link. Whenever a vertex is moved, updates on the congHeap and commTasks are performed for all the incoming and outgoing edges of $t_{m c}$ and $t$ in $E_{t}$. If there is no improvement after $\Delta=8$ trials, the early exit mechanism terminates the inner for loop. Then, the next task in commTasks $\left[e_{m c}\right]$ is chosen and the search restarts. If no improvement is found for the most congested link the algorithm stops. This algorithm can be applied both to coarser and finer task graphs. However, we only apply it on the coarser graph due to the reasons explained before.

With static routing, a message route can be found in $\mathcal{O}(D)$ time. The congestions of all the links can be calculated in $\mathcal{O}\left(D\left|E_{t}\right|\right)$ time, and the cost of initializing congHeap is $\mathcal{O}\left(\left|E_{m}\right| \log \left|E_{m}\right|\right)$. A task insertion to a commTasks set (implemented as a red-black binary tree using std:set in $\mathrm{C}++)$ can be done in $\mathcal{O}\left(\log \left|V_{t}\right|\right)$. Since each message (an edge in $E_{t}$ ) can pass through at most $D$ links, the complexity of commTasks's initialization is $\mathcal{O}\left(D\left|E_{t}\right| \log \left|V_{t}\right|\right)$. Therefore the initialization phase has a complexity of $\mathcal{O}\left(D\left|E_{t}\right| \log \left|V_{t}\right|+\left|E_{m}\right| \log \left|E_{m}\right|\right)$. A refinement pass starts at line 2. The main for loop iterates at most $\left|V_{t}\right|$ times and the complexity of a BFS at line 3 is $\mathcal{O}\left(\left|E_{m}\right|\right)$. Hence, the overall BFS complexity in a pass is $\mathcal{O}\left(\left|V_{t}\right|\left|E_{m}\right|\right)$. For each candidate swap operation, we compute MC and AC by temporarily updating congHeap where an update costs $\log \left|E_{m}\right|$ and a candidate swap requires at most $D$ updates for each of the $t_{m c}$ and $t$ edges. Since we consider at most $\Delta$ swap operations for a $t_{m c}$ which can be any vertex in $V_{t}$, the cost of MC and AC computation is $\mathcal{O}\left(E_{t} \Delta D \log \left|E_{m}\right|\right)$ for each $e_{m c}$. Once an improvement is found, the data structures congHeap and commTasks are updated and this happens only once per pass (a while loop iteration). Hence, the overall complexity of a pass is dominated by the BFS and MC-AC computations. Therefore the overall complexity of a pass becomes $\mathcal{O}\left(\left(E_{t} \Delta D \log \left|E_{m}\right|\right)+\left(\left|V_{t}\right|\left|E_{m}\right|\right)\right)$.

Algorithm 3 accurately sees the maximum congestion on a static-routing network. For the networks with dynamic routing, an approximate refinement algorithm with a similar structure 
can be used. For example, the bandwidth on the Blue Gene/Q and Blue Gene/P can be maximized by placing the heavily communicating tasks to the diagonals of the torus [22], [23].

\section{EXPERIMENTS}

In order to evaluate the quality of the mapping algorithms, we conducted various experiments on two irregular applications, an SpMV kernel and a synthetically generated application. The proposed methods are implemented in the UMPA framework. The $\mathrm{U}_{\mathrm{G}}$ and $\mathrm{U}_{\mathrm{wH}}$ variants minimize $\mathrm{WH}$ using Algorithms 1 and 2, and $\mathrm{U}_{\mathrm{MC}}$ and $\mathrm{U}_{\mathrm{MMC}}$ minimizes $\mathrm{MC}$ and MMC, respectively using Algorithm 3 (we do not give the results for $\mathrm{TH}$ variant as they are very close to those of $\mathrm{U}_{\mathrm{G}}$ and $\mathrm{U}_{\mathrm{WH}}$ ). These mapping methods are compared against the default MPI mapping (SMP-STYLE) in Hopper (DEF), the mapping provided by SCOTCH (SMAP, version 5.1.0 as the newer one does not support sparse allocations) [1], and the ones provided by LibTopoMap (TMAP) [15].

We selected 25 matrices from University of Florida (UFL) sparse matrix collection, belonging to 9 different classes (the list is at the supplementary page: http://web.cse.ohio-state.edu/ deveci/umpamap). We used 7 graph and hypergraph partitioners to partition these matrices: Scotch [1], KaffPa (Kahip) [2], Metis [3], Рatoh [4], and UMPA [5]. A summary of the partitioning results are given in Section IV-A. MPI task communication graphs corresponding to these partitions are created and mapped to real processor allocations in Hopper. The analysis of the metrics and algorithm efficiency is presented in Section IV-B. Section IV-C analyzes the impact of the mapping algorithms on the communication time, whereas Section IV-D evaluates the performance improvements for a Trilinos SpMV kernel [28]. We analyze the impact of the partitioning and mapping metrics on the parallel performance in Section IV-E.

\section{A. Partitioning results}

The matrices are first converted to a column-net hypergraph model, i.e., the rows represent the tasks with loads proportional to their number of non-zeros. The columns represent sets of data communications where each message has a unit communication costs. On these matrices we perform 1D row-wise partitioning for 1024, 2048, 4096, 8192 and 16384 parts (we only use 19 matrices for 16384 parts; balanced partitions were not feasible for the remaining 6). The graph partitioners, $\mathrm{SCOTCH}$ and KAFFPA, are run to minimize the edge-cut, and METIS and $\mathrm{PATOH}$ are run to minimize the total communication volume TV. Being a multi-objective partitioner, UMPA is used with different metrics: $\mathrm{UMPA}_{\mathrm{MV}}$ minimizing maximum send volume (MSV) and TV; $\mathrm{UMPA}_{\mathrm{MM}}$ minimizing maximum number of sent messages (MSM), total number of messages (TM) and TV; $\mathrm{UMPA}_{\mathrm{TM}}$ minimizing TM and TV; as their primary, secondary, and tertiary objectives, respectively [5]. All the partitioners are run with their default parameters.

Figure 1 shows the mean metric values normalized with that metric value of РАТОН. Overall, all the tools obtain similar results, but edge-cut minimizing ones, SСOTCH and KAFFPA, obtain a slightly worse communication volume quality. For the MSV metric, UMPA $\mathrm{MV}_{\mathrm{M}}$ has the best results, e.g., it obtains a $5-10 \%$ better average MSV value w.r.t. РATOH which obtains the best results for TV. For the message metrics, UMPAMM obtained a 16-19\% better MSM value, and UMPA ${ }_{\text {TM }}$ obtained a $9-10 \%$ better TM value. These numbers are not given here to compare the partitioners since the experiment is not designed for that purpose. We want to better understand the impact of the partitioning and mapping metrics on the execution time.

\section{B. Mappings on Hopper}

Here we evaluate the mapping metric results on Hopper, which has a 3D Torus network and 24 processors per node. Even though the proposed algorithms do not have constraints on the number of processors, we tested them on numbers that are powers of two. Using all the processors in a node results in non-uniform processor allocations per node (since 24 does not divide 1024), in which case we experienced a few failing algorithms in LibTopoMap. Therefore, we used 16 processors per node (4 processors per NUMA domain).

We create directed task graphs by running all the partitioners on each matrix; for each graph and part number, we have 7 MPI task graphs. We will refer a task graph as $G_{t}^{\mathrm{X}}$ when the part vector obtained from the partitioner $\mathrm{X}$ is used to create it. The mapping algorithms are then used to map these graphs to 5 different Hopper processor allocations. Figure 2 shows the average metric values of all mapping algorithms normalized to those of the default mapping on $G_{t}^{\mathrm{PATOH}}$ graphs. Almost all algorithms have their best WH and MC values on $G_{t}^{\text {PATOH }}$, and best TH and MMC values on $G_{t}^{\text {UMPAтм }}$. The results are expected for $\mathrm{WH}$ and $\mathrm{TH}$, since $\mathrm{WH}$ is closely related to the communication volume, and $\mathrm{TH}$ is related to total number of messages. On the other hand, it is expected to have better MC and MMC values on the task graphs with lower MSV and MSM values, respectively. However, in our experiments, we see a better correlation of these metrics with TV and TM.

In Fig. 2, the DEF mapping obtains already good results on WH and TH. This is due to the part ID assignment in recursivebisection-based partitioners and the placement mechanism in Hopper: the partitioner puts highly communicating tasks to the parts with closer IDs. On the machine side, Hopper places the consecutive MPI ranks within a single node, then it moves to the closer nodes using space filling curves. Therefore, highly communicating consecutive MPI ranks are placed fairly close to each other. However, there is still room for improvement when we exploit the actual task communication requirements. For example, $\mathrm{U}_{\mathrm{G}}$ obtains $5-18 \%$ and $5-17 \%$ better values on $\mathrm{WH}$ and $\mathrm{TH}$, respectively.

Metric improvements on more sparse allocations (with less number of processors) are higher: $\mathrm{U}_{\mathrm{G}}$ significantly reduces WH and $\mathrm{TH}$, and $\mathrm{U}_{\mathrm{wH}}$ improves them by another $4-5 \%$. Also the variants that improve the WH metric also improve MC and MMC. For example, $\mathrm{U}_{\mathrm{G}}\left(\mathrm{U}_{\mathrm{wH}}\right)$ improves MC by $4 \%(10-12 \%)$ on 1024 and 2048 processors. However, when the number of processors is high, they increase the MC metric by $13-36 \%$ when the number of parts is high. Still, they reduce MMC, 


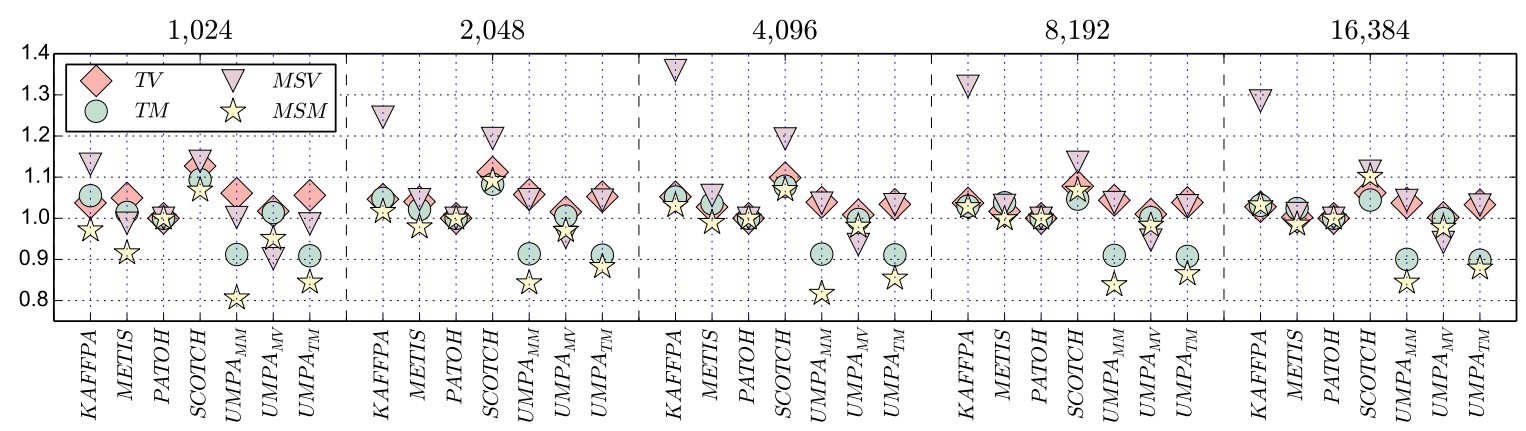

Fig. 1: Geometric means of the partition metrics w.r.t PATOH for the corresponding part number.

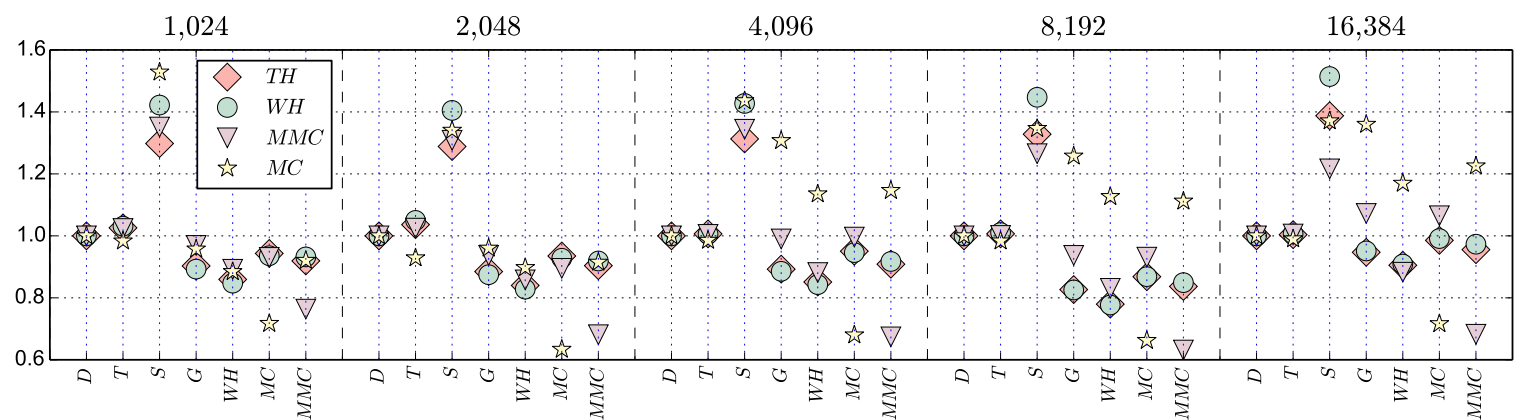

Fig. 2: Mean metric values of the algorithms on $G_{t}^{\mathrm{P} \text { Атон }}$ graphs normalized w.r.t. those of DEF. The numbers at the top denote the number of the processors, and the letters at the bottom correspond to the mapping algorithms DEF, TMAP, SMAP, $\mathrm{U}_{\mathrm{G}}, \mathrm{U}_{\mathrm{WH}}, \mathrm{U}_{\mathrm{MC}}$, $\mathrm{U}_{\mathrm{MMC}}$, respectively.

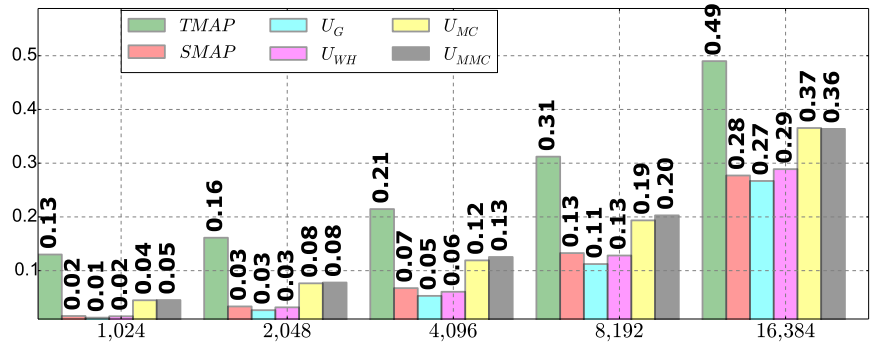

Fig. 3: (Geometric) mean execution times of different mapping algorithms on PATOH partitions. The time of $\mathrm{U}_{\mathrm{wH}}, \mathrm{U}_{\mathrm{MC}}$, and $\mathrm{U}_{\mathrm{MMC}}$ includes $\mathrm{U}_{\mathrm{G}}$ time, as they run on top of it.

$\mathrm{TH}$, and $\mathrm{WH}$ except $\mathrm{U}_{\mathrm{G}}$ on 16,384 processors. Also, $\mathrm{U}_{\mathrm{MC}}$ significantly reduces $(27-37 \%)$ the MC metric for all cases and have $1-13 \%$ improvement on $\mathrm{WH}$ and $\mathrm{TH}$. Similarly, $\mathrm{U}_{\mathrm{MMC}}$ reduces MMC by $24-37 \%$ with small increases on $\mathrm{TH}$ and $\mathrm{WH}$.

LibTopoMap provides six algorithms, and the best one in our experiments employs recursive graph bi-partitioning. Here we only present the best variant's performance (TMAP or T). The primary metric for LibTopoMap is MC. If TMAP's MC value is not smaller than the DEF mapping, it returns the DEF mapping. Overall, TMAP improves MC by $1-7 \%$ with $1-5 \%$ increase on the other metrics. On the other hand, SMAP's results are worse than DEF mappings for most of the cases.

Figure 3 presents the (geometric) mean mapping times of the algorithms. The times of the SMAP, $U_{G}$ and $U_{W H}$ are the lowest, and they are followed by $\mathrm{U}_{\mathrm{MC}}$ and $\mathrm{U}_{\mathrm{MMC}}$. TMAP's execution time is more than the other methods, and it is 1.3-2.6 times slower than the slowest UMPA variant.

\section{Communication-only experiments}

In task mapping, the communication is usually modeled by assuming all the messages are transferred at once. However, this may not be the case in practice: load imbalance can delay some transfers, and applications might be using common techniques such as communication-computation overlap to hide the latency. Hence, improvements due to mapping may not be visible on an application's execution time. Here, to limit the impact of these factors, we generate irregular, communicationonly applications based on the SpMV communication patterns of the two largest matrices in our dataset: cage15 and rgg_n_2_23_s0 (in short rgg). In this SpMV-like executions, no computation is performed, and all the transfers are initialized at the same time where each processor follows the pattern in the corresponding communication graph. Therefore the total execution time of this application is equal to its communication time. To make the improvements more visible and reduce the noise, we scale the message sizes by using the factors $4 K$ and $256 K$ for cage 15 and $r g g$, respectively. The experiment is performed with 4096 processors. Each mapping algorithm is run with the 7 communication graphs (one per partitioner), and for each mapping, the execution is repeated 5 times to reduce the noise on the time. Figure 4 shows the normalized mean execution times with standard deviations and the metric values ( In real case, link congestions are also affected by the other running jobs. The reported congestion-metrics refer to those only incurred by the application.) normalized w.r.t. those of DEF mapping on $G_{t}^{\text {PATOH }}$. Although we run SMAP in this experiment, its communication time is worse than the others (we exclude SMAP from the figure for clarity). We do not report TH, as it is highly correlated with WH. Results with 8192 processors and a different sparse allocation can be found at http://web.cse.ohio-state.edu/ deveci/umpamap.

Figure $4 \mathrm{a}$ shows the results for cage15 communication 


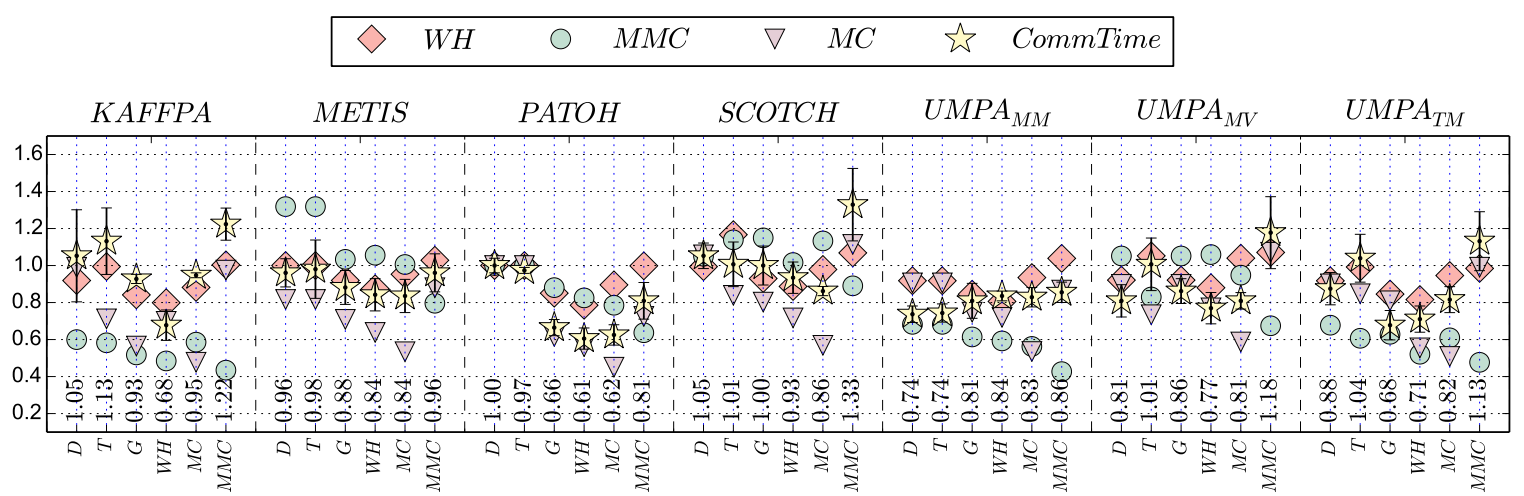

(a) cage 15, \#procs $=4096$, scale $=4 \mathrm{~K}$

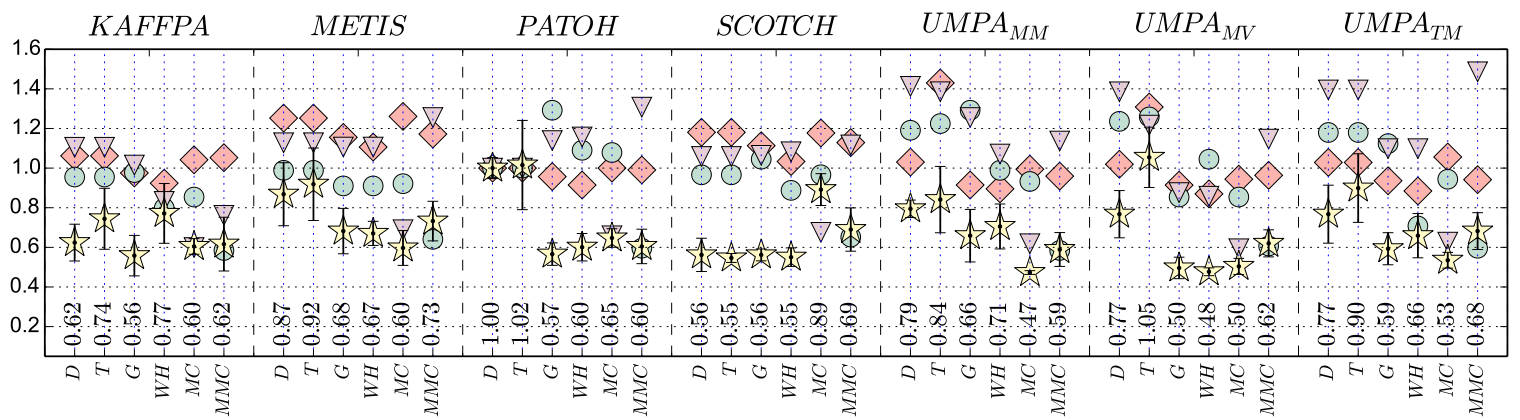

(b) rgg, \#procs $=4096$, scale $=256 \mathrm{~K}$

Fig. 4: Average execution times and metrics for pure communication-based applications generated from cage 15 and $r g g$ : the numbers at the bottom are the normalized execution times w.r.t. DEF mapping on $G_{t}^{\mathrm{PATOH}}$. The partitioner names are given at the top, and the names at the bottom are the mapping algorithms, as given in Figure 2.

graphs. The overall execution time correlates well with wH. In most cases, $\mathrm{U}_{\mathrm{G}}$ and $\mathrm{U}_{\mathrm{WH}}$ improve $\mathrm{WH}, \mathrm{MC}$, and the communication time w.r.t. DEF with a few exceptions. For example, on $G_{t}^{\mathrm{UMPA}_{\mathrm{MM}}}$, wH minimizing algorithms (Algorithms 1 and 2) improve all three metrics at the same time. However, the execution times with these mappings slightly increase. $\mathrm{U}_{\mathrm{wH}}$ obtains much better WH, MC, and execution times compared to $\mathrm{U}_{\mathrm{G}}$. On $G_{t}^{\mathrm{KAFPPA}}$, it improves $\mathrm{WH}$ in the expense of increasing MC but the execution time significantly reduces. Overall, $\mathrm{U}_{\mathrm{G}}$ and $\mathrm{U}_{\mathrm{wH}}$ improve the performance up to $34 \%$ and $39 \%$ w.r.t. the DEF. For all graphs, $\mathrm{U}_{\mathrm{MC}}$ obtains the best MC values and it usually improves the performance w.r.t. DEF. Moreover, it obtains the best execution time on $G_{t}^{\mathrm{SCOTсH}}$, which has the highest TV w.r.t other partitioners on this graph. Among the UMPA algorithms, $U_{\text {ммс }}$ obtains the worst execution times although it always significantly reduces MMC. This is expected; since the message sizes are scaled, the executions have a high TV value and the volume-related metrics are likely to be the bottleneck rather than the message-related ones. TMAP can not improve the results of the DEF in some of cases, e.g., $G_{t}^{\text {METIS }}, G_{t}^{\text {РАTоH }}$, and $G_{t}^{\text {UMPAмм }}$, and returns the default mapping (the times vary $2-3 \%$ due to noise). Lastly, although DEF obtains the best mean execution time on UMPA graphs, overall, the best times are obtained on РATOH graphs with $\mathrm{U}_{\mathrm{WH}}$ and $\mathrm{U}_{\mathrm{MC}}$ with $39 \%$ and $38 \%$ improvement, respectively.

Figure $4 \mathrm{~b}$ shows the results for rgg communication graphs. The proposed mapping algorithms improve the execution time

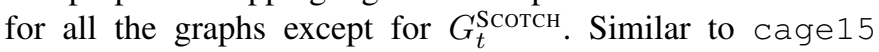

experiments, the best performance is obtained by $\mathrm{U}_{\mathrm{G}}, \mathrm{U}_{\mathrm{MC}}$ and $\mathrm{U}_{\mathrm{WH}}$. The best execution time is achieved by $\mathrm{U}_{\mathrm{MC}}$ on $G_{t}^{\text {UMPAмM }}$ with a $40 \%$ improvement w.r.t DEF mapping. TMAP obtains the same mappings with DEF on most of the graphs except $G_{t}^{\text {UMPамм }}$ and $G_{t}^{\text {UMPAMv }}$. As the results for $G_{t}^{\text {PAтон }}$ show, the proposed algorithms improves the performance $35-43 \%$ for rgg experiments.

The execution time is improved better with the algorithms minimizing WH and then MC. The improvements achieved by $\mathrm{U}_{\text {MMC }}$ is not as high as the others since for these "scaled" applications, the volume metrics are likely to be the bottleneck. In Section IV-E, we perform a regression analysis to better analyze the relation between the metrics and the execution time.

\section{SpMV experiments}

In this section, we study the impact of the proposed algorithms on the SpMV performance. We use cage 15 and perform SpMV using the Tpetra package of Trilinos with 500 and 1000 iterations. Figure 5 shows the performance results, where each metric and the overall execution time is normalized w.r.t. that of DEF on $G_{t}^{\mathrm{PATOH}}$. The experiment is run for 4096 and 8192 processors on two different allocations. Only the results of a single allocation on 4096 processors is shown due to space limitations. The rest of results can be found at the supplementary page. The SpMV operation is repeated 5 times for each mapping and communication graph. We report the average of these 5 executions, and error bars represent the standard deviations. Unlike the previous experiment, TH is reported instead of $\mathrm{WH}$, as its correlation with the total execution time is better. 


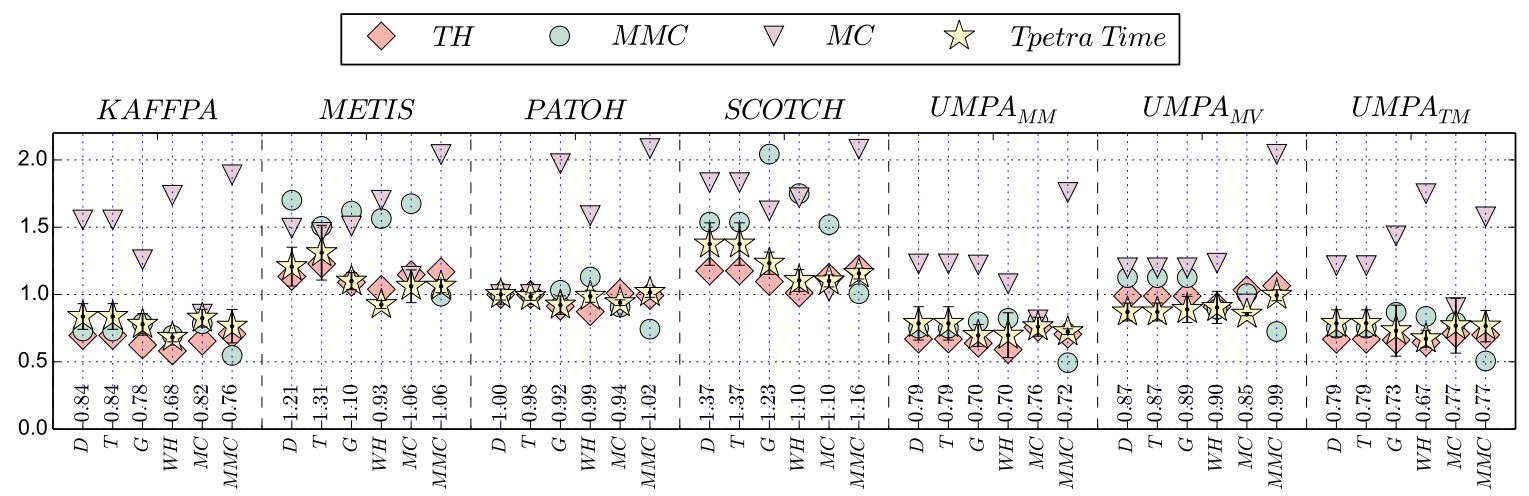

Fig. 5: Trilinos SpMV results for cage15 on 4096 processors. Each metric is normalized w.r.t that of DEF on $G_{t}^{\text {PAтон }}$.

In this setting, $\mathrm{U}_{\mathrm{WH}}$ obtains the best performance; it decreases the overall execution time almost always (except for $G_{t}^{\mathrm{UMPAMv}}$ ) and up to $23 \%$ (for $G_{t}^{\mathrm{METIS}}$ ) w.r.t DeF. $\mathrm{U}_{\mathrm{G}}$ obtains a similar performance with slightly higher execution times. Although $\mathrm{U}_{\mathrm{MC}}$ improves the performance for many cases w.r.t DEF, its performance is not as competitive as in the previous experiment since the message sizes are much smaller. Similar to the communication-only experiment, $\mathrm{U}_{\mathrm{MMC}}$ obtains smaller improvements than the other UMPA variants. The overall performance of TMAP is very close to DEF, since it returns the DEF mapping for most of the cases.

Overall, TH highly correlates with the execution time. Moreover, this correlation also holds among different communication graphs. In Section IV-B, we already observed that $\mathrm{TH}$ is much lower on the graphs with a lower TM. Improving the TH metric via both partitioning (with the objective TM) and mapping significantly reduces the parallel SpMV time. The best TM values for cage 15 have been found by KAFFPA, $\mathrm{UMPA}_{\mathrm{MM}}$ and $\mathrm{UMPA}_{\mathrm{TM}}$ (see the supplementary page for a cage 15-only version of Fig. 1) and as Fig. 5 shows, these are the best partitioners for the default mapping. $U_{\mathrm{WH}}$ reduces the execution time by another $9-16 \%$ for the for these cases and obtains the best overall execution time for $G_{t}^{\mathrm{UMPATM}}$. This is more than two times faster than the slowest variant, which is obtained by the DEF on $G_{t}^{\mathrm{SCOTCH}}$. It also has $34 \%$ lower TM and $44 \%$ lower TH value. This shows the importance both the partitioning and mapping on SpMV performance.

\section{E. Regression analysis}

To analyze the performance improvements obtained for the communication-only applications and SpMV kernel w.r.t. the partitioning and mapping metrics, we use a linear regression analysis technique and solve a nonnegative least squares problem (NNLS). In NNLS, given a variable matrix $\mathbf{V}$ and a vector $\mathbf{t}$, we want to find a dependency vector $\mathbf{d}$ which minimizes $\|\mathbf{V d}-\mathbf{t}\|$ s.t. $\mathbf{d} \geq 0$. In our case, V has 14 columns: the partitioning metrics MSV, TV, MSM, TM; the mapping metrics $\mathrm{WH}, \mathrm{TH}, \mathrm{MC}, \mathrm{MMC}, \mathrm{AC}$, AMC; inter-node communication volume (ICV), i.e., the total communication volume on the network excluding the intra-node communication (from TV); number of inter-node communication messages (ICM); the maximum receive volume of a node (MNRV); and the maximum number of messages received by a node (MNRM). A row $\mathbf{t}$ of $\mathbf{V}$ corresponds to an execution where the time is put to the corresponding entry of $\mathbf{t}$. To standardize each entry of $\mathbf{V}$ and make them equally important, each column of $\mathbf{V}$ is normalized by first subtracting the column mean from each column entry, and dividing them to the column standard deviation. We then use MATLABs lsqnonneg to solve NNLS. The coefficient $\mathbf{d}_{i}$ of the output shows the dependency of the execution time to the $i$ th metric.

We perform linear regression on the communication-only experiments' results with cage15 graphs, 4096 processors and two sparse Hopper allocations. The analysis distinguished three metrics with non-zero coefficients. The metric with the highest coefficient is WH, followed by MSV and MC (0.023, 0.020 and 0.20 ), whereas the message-based metrics are found not to highly correlate with the performance. This is expected since the communication is scaled and the volume metrics' importance are increased. The results show that from the mapping perspective, $\mathrm{WH}$ and $\mathrm{MC}$ are the most important metrics for the applications with a high communication volume, whereas from the partitioning perspective, it is likely to be MSV.

We used the same experimental setting (cage 15, 4096 processors, two allocations) for the SpMV kernel which is more latency bounded than the communication-only counterpart since there is no scaling on the communication volume. The metrics with non-zero coefficients are found to be AMC, ICV, MMC, TH, and MNRV (0.109, 0.070, 0.051, 0.050, 0.040). Since AMC better correlates with the performance compared to $\mathrm{TH}$, it can be a good practice to utilize the already used links while reducing TH. One weakness of the regression analysis is that when highly correlating metrics are given in $\mathbf{V}$, the analysis may return a positive coefficient for only one of them. In our case, the importance of MNRM, ICM, and TM is hidden by the regression analysis. We also computed pairwise Pearson correlation of the metrics and observed a high correlation $(\geq 0.92)$ of these metrics with AMC.

\section{F. Summary}

Table I presents a summary of the improvements achieved by the mapping algorithms in our experiments. For each allocation and part number, we calculate the geometric mean of the execution times obtained with the mapping methods on all graphs. The table shows the geometric mean of the execution times for DEF, and the normalized time for the 
TABLE I: Average improvements of the mapping algorithms on communication-only applications and SpMV kernel that runs for 500 and 1000 iterations for the first and second allocations, respectively.

\begin{tabular}{|c|c|c|c|c|c|c|c|c|}
\hline & \# procs & Rep. & DEF & TMAP & $\mathrm{U}_{\mathrm{G}}$ & $\mathrm{U}_{\mathrm{WH}}$ & $\mathrm{U}_{\mathrm{MC}}$ & $\mathrm{U}_{\mathrm{MMC}}$ \\
\hline \multirow{5}{*}{$\begin{array}{l}n \\
\overrightarrow{0} \\
0 \\
0 \\
0 \\
0\end{array}$} & \multirow{2}{*}{4096} & 1 & $1.44 \mathrm{sec}$. & 1.01 & 0.93 & 0.87 & 0.93 & 0.95 \\
\hline & & 2 & $2.77 \mathrm{sec}$. & 1.00 & 0.91 & 0.89 & 0.94 & 0.95 \\
\hline & \multirow{3}{*}{8192} & 1 & $1.25 \mathrm{sec}$. & 0.99 & 0.98 & 0.96 & 0.99 & 1.01 \\
\hline & & 2 & $3.43 \mathrm{sec}$. & 1.01 & 0.99 & 0.94 & 1.01 & 1.04 \\
\hline & & Gmean & $2.03 \mathrm{sec}$. & 1.00 & 0.95 & 0.91 & 0.97 & 0.99 \\
\hline \multirow{5}{*}{ 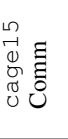 } & \multirow{2}{*}{4096} & 1 & $0.28 \mathrm{sec}$. & 1.06 & 0.90 & 0.83 & 0.88 & 1.15 \\
\hline & & 2 & $0.28 \mathrm{sec}$. & 1.06 & 0.88 & 0.82 & 0.88 & 1.18 \\
\hline & \multirow{3}{*}{8192} & 1 & 0.19 sec. & 1.01 & 1.02 & 0.89 & 1.01 & 1.16 \\
\hline & & 2 & $0.20 \mathrm{sec}$. & 1.00 & 0.95 & 0.89 & 0.99 & 1.18 \\
\hline & & Gmean & 0.23 sec. & 1.03 & 0.93 & 0.86 & 0.94 & 1.17 \\
\hline \multirow{3}{*}{ 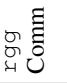 } & \multirow{3}{*}{4096} & 1 & 0.39 sec. & 1.11 & 0.77 & 0.83 & 0.79 & 0.85 \\
\hline & & 2 & 0.33 sec. & 0.98 & 0.96 & 0.77 & 0.84 & 0.87 \\
\hline & & Gmean & $0.36 \mathrm{sec}$. & 1.05 & 0.86 & 0.80 & 0.81 & 0.86 \\
\hline
\end{tabular}

other algorithms. The average for all allocations and part numbers are given at the bottom of the table. Overall, $U_{\mathrm{wH}}$ improves the cage 15 SpMV kernel time by 4-13\%, whereas the improvements for the communication-only cage 15 and rgg applications are $14 \%$ and $20 \%$, respectively, w.r.t. DEF.

\section{CONCLUSION}

We have proposed fast and high quality topology-aware task mapping methods that use graph models. We have compared the proposed methods with some other graph-based algorithms from the literature and with a default method used in Nersc's Hopper Supercomputer. The experiments showed that on a set of 25 matrices from the UFL collection, the proposed methods obtained high quality mappings in a very short time for the target system. The experiments with 4096 processors revealed significant improvements on the mapping metrics compared to the Hopper's default mapping. These improvements yield a $43 \%$ performance improvement on one case for a communication-only application and a $23 \%$ improvement on the SpMV performance. Overall, with 4096 and 8192 processors, the proposed algorithms improve the performance of the SpMV kernel and the communication-only applications by $9 \%$ and $14 \%$, respectively. We also evaluated the metrics according to their correlation with the performance. For the applications with a large communication volume, our analysis revealed that the weighted hop metric is the most dominant one, and for those with smaller messages, the average message congestion is a good metric that correlates with the performance.

\section{ACKNOWLEDGMENT}

This work was supported in parts by the DOE grant DEFC02-06ER2775; by the NSF grants CNS-0643969, OCI0904809 and OCI-0904802; and by France ANR project SOLHAR (ANR-13-MONU-0007).

\section{REFERENCES}

[1] F. Pellegrini and J. Roman, "Scotch: A software package for static mapping by dual recursive bipartitioning of process and architecture graphs," in High-Performance Computing and Networking. Springer, 1996, pp. 493-498.

[2] P. Sanders and C. Schulz, "Engineering multilevel graph partitioning algorithms," in Algorithms-ESA 2011. Springer, 2011, pp. 469-480.

[3] G. Karypis, MeTiS: A software package for partitioning unstructured graphs, partitioning meshes, and computing fill-reducing orderings of sparse matrices version 5.0, University of Minnesota, Department of Comp. Sci. and Eng., Army HPC Research Center, 2011.
[4] Ü. V. Çatalyürek and C. Aykanat, PaToH: A Multilevel Hypergraph Partitioning Tool, Version 3.0, Bilkent University, Department of Computer Engineering, 1999.

[5] M. Deveci, K. Kaya, B. Uçar, and Ü. V. Çatalyürek, "Hypergraph partitioning for multiple communication cost metrics: Model and methods," Journal of Parallel and Distributed Computing (to appear), 2014.

[6] H. M. Aktulga, C. Yang, E. G. Ng, P. Maris, and J. P. Vary, "Topologyaware mappings for large-scale eigenvalue problems," in Euro-Par 2012 Parallel Processing. Springer, 2012, pp. 830-842.

[7] H. Kikuchi, B. Karki, and S. Saini, "Topology-aware parallel molecular dynamics simulation algorithm," in Intl Conf Parallel \& Distributed Proc Tech \& Applications, 2006.

[8] A. Bhatele, L. V. Kale, and S. Kumar, "Dynamic topology aware load balancing algorithms for molecular dynamics applications," in $23 \mathrm{rd}$ Intl Conf Supercomputing. ACM, 2009, pp. 110-116.

[9] W. M. Brown, T. D. Nguyen, M. Fuentes-Cabrera, J. D. Fowlkes, P. D. Rack, M. Berger, and A. S. Bland, "An evaluation of molecular dynamics performance on the hybrid Cray XK6 supercomputer," in Intl Conf Computational Science (ICCS), 2012.

[10] F. Gygi, E. W. Draeger, M. Schulz, B. de Supinski, J. Gunnels, V. Austel, J. Sexton, F. Franchetti, S. Kral, C. Ueberhuber, and J. Lorenz, "Large-scale electronic structure calculations of high-Z metals on the BlueGene/L platform," in ACM/IEEE Conf Supercomputing, 2006.

[11] G. Almasi, S. Chatterjee, A. Gara, J. Gunnels, M. Gupta, A. Henning, J. Moreira, and B. Walkup, "Unlocking the performance of the BlueGene/L supercomputer," in ACM/IEEE Conf Supercomputing, 2004.

[12] H. Yu, I.-H. Chung, and J. Moreira, "Topology mapping for Blue Gene/L supercomputer," in ACM/IEEE Conf Supercomputing, 2006

[13] J. A. Pascual, J. Miguel-Alonso, and J. A. Lozano, "Optimization-based mapping framework for parallel applications," Journal of Parallel and Distributed Computing, vol. 71, no. 10, pp. 1377-1387, 2011

[14] M. Deveci, S. Rajamanickam, V. Leung, K. T. Pedretti, S. L. Olivier, D. P. Bunde, Ü. V. Çatalyürek, and K. D. Devine, "Exploiting geometric partitioning in task mapping for parallel computers," in 28th IPDPS, Phoenix, AZ, 2014

[15] T. Hoefler and M. Snir, "Generic topology mapping strategies for largescale parallel architectures," in 25th ACM Supercomputing, 2011.

[16] A. Bhatele, G. Gupta, L. Kale, and I.-H. Chung, "Automated mapping of regular communication graphs on mesh interconnects," in Intl Conf High Performance Computing, 2010.

[17] S. W. Bollinger and S. F. Midkiff, "Heuristic technique for processor and link assignment in multicomputers," IEEE Trans Comput, vol. 40, no. 3, pp. 325-333, 1991.

[18] I.-H. Chung, C.-R. Lee, J. Zhou, and Y.-C. Chung, "Hierarchical mapping for HPC applications," in Workshop Large-Scale Parallel Processing, 2011, pp. 1810-1818.

[19] T. Chockalingam and S. Arunkumar, "Genetic algorithm based heuristics for the mapping problem," Computers and Operations Research, vol. 22, no. 1, pp. 55-64, 1995.

[20] C. Walshaw and M. Cross, "Multilevel mesh partitioning for heterogeneous communication networks," Future generation computer systems, vol. 17 , no. 5, pp. 601-623, 2001.

[21] F. Pellegrini and J. Roman, "Experimental analysis of the dual recursive bipartitioning algorithm for static mapping," LaBRI, URA CNRS 1304, Univ. Bordeaux I, Tech. Rep. TR 1038-96, 1996.

[22] A. Bhatele, T. Gamblin, S. H. Langer, P. Bremer, E. W. Draeger, B. Hamann, K. E. Isaacs, A. G. Landge, J. A. Levine, V. Pascucci, M. Schulz, and C. H. Still, "Mapping applications with collectives over sub-communicators on torus networks," in High Performance Computing, Networking, Storage and Analysis (SC), 2012. IEEE.

[23] A. Bhatele, N. Jain, K. E. Isaacs, R. Buch, T. Gamblin, S. H. Langer, and L. V. Kale, "Optimizing the performance of parallel applications on a 5D torus via task mapping," in IEEE International Conference on High Performance Computing. IEEE Computer Society, Dec. 2014.

[24] S. H. Bokhari, "On the mapping problem," IEEE Trans Comput, vol. 100 , no. 3, pp. 207-214, 1981.

[25] C. Albing, N. Troullier, S. Whalen, R. Olson, and J. Glensk, "Topology, bandwidth and performance: A new approach in linear orderings for application placement in a 3D torus," in Cray User Group (CUG), 2011.

[26] C. M. Fiduccia and R. M. Mattheyses, "A linear-time heuristic for improving network partitions," in 19th Design Automation Conf., 1982.

[27] B. Kernighan and S. Lin, "An efficient heuristic procedure for partitioning graphs," The Bell System Technical Journal, Feb 1970.

[28] M. A. Heroux, R. A. Bartlett, V. E. Howle, R. J. Hoekstra, J. J. Hu, T. G. Kolda, R. B. Lehoucq, K. R. Long, R. P. Pawlowski, E. T. Phipps et al., "An overview of the trilinos project," ACM Transactions on Mathematical Software (TOMS), vol. 31, no. 3, pp. 397-423, 2005. 